\title{
The effects of a 4-week mesocycle of barbell back squat or barbell hip thrust strength training upon isolated lumbar extension strength
}

\author{
Alexander Hammond ${ }^{1}$, Craig Perrin ${ }^{1}$, James Steele ${ }^{1}$, Jürgen Giessing ${ }^{2}$, Paulo Gentil ${ }^{3}$, James P Fisher ${ }^{\text {Corresp. } 1}$ \\ ${ }^{1}$ School of Sport, Health and Social Sciences, Southampton Solent University, Southampton, UK \\ 2 Institute of Sport Science, Universität Koblenz-Landau, Landau, Germany \\ 3 Faculty of Physical Education and Dance, Universidade Federal de Goiás, Goiania, Brazil \\ Corresponding Author: James P Fisher \\ Email address: james.fisher@solent.ac.uk
}

Objectives: Common exercises such as the barbell back squat (BBS) and barbell hip thrust $(\mathrm{BHT})$ are perceived to provide a training stimulus to the lumbar extensors. However, to date there have been no empirical studies considering changes in lumbar extension strength as a result of BBS or BHT resistance training interventions. Purpose: To consider the effects of barbell back squat (BBS) and barbell hip thrust (BHT) resistance training programmes upon isolated lumbar extension (ILEX) strength. Methods: Trained male subjects $(n=14 ; 22.07 \pm 0.62$ years; $179.31 \pm 6.96 \mathrm{~cm} ; 79.77 \pm 13.81 \mathrm{~kg}$ ) were randomised in to either BBS $(n=7)$ or $\mathrm{BHT}(n=7)$ groups and performed 2 training sessions per week during a 4-week mesocycle using $80 \%$ of their 1RM. All subjects were tested preand post-intervention for BBS and BHT 1RM as well as isometric ILEX strength. Results: Analyses revealed that both BBS and BHT groups significantly improved both their BBS and BHT 1RM, suggesting a degree of transferability. However, the BBS group improved their BBS 1RM to a greater degree than the BHT group $(p=0.050 ; \sim 11.8 \mathrm{~kg} / 10.2 \%$ vs. $\sim 8.6 \mathrm{~kg} / 7.7 \%$, respectively). And the BHT group improved their BHT 1RM to a greater degree than the BBS group ( $p=0.034 ; \sim 27.5 \mathrm{~kg} / 24.8 \%$ vs. $\sim 20.3 \mathrm{~kg} / 13.3 \%$, respectively). Neither BBS nor BHT groups significantly improved their isometric ILEX strength.

Conclusions: The present study supports the concept of specificity, particularly in relation to the movement mechanics between trunk extension (including pelvic rotation) and ILEX. Our data suggests that strength coaches, personal trainers, and trainees can self-select multi-joint lower body trunk extension exercises based on preference or variety. However, evidence suggests that neither the BBS nor BHT exercises can meaningfully increase isolated lumbar extension strength. Since strengthening these muscles might enhance physical and sporting performance we encourage strength coaches and personal trainers to prescribe ILEX exercise.

Peer) reviewing PDF | (2019:03:35781:2:0:NEW 12 Jun 2019) 
1 The effects of a 4-week mesocycle of barbell back squat or barbell hip thrust strength training upon

2 isolated lumbar extension strength

3

4 Running Title: Back squat and Hip Thrust for low-back strength

5

6 Original research article

$7 \quad$ Alexander Hammond ${ }^{1}$, Craig Perrin ${ }^{1}$, James Steele ${ }^{1}$, Jürgen Giessing ${ }^{2}$, Paulo Gentil ${ }^{3}$, James P Fisher ${ }^{1}$

$8{ }^{1}$ School of Sport, Health and Social Sciences, Southampton Solent University, Southampton, UK

92 Institute of Sport Science, Universität Koblenz-Landau, Landau, Germany

$10{ }^{3}$ Faculty of Physical Education and Dance, Universidade Federal de Goiás, Goiania, Brazil

11

12 Corresponding Author:

13 James Peter Fisher

14 School of Sport, Health and Social Sciences

15 Southampton Solent University

16 East Park Terrace

17 Southampton

18 UK

19 Tel: +44 2382013163

20 Email: james.fisher@solent.ac.uk

21

22 Co-Author contact details:

23 Alexander Hammond (alex.hammond01@btinternet.com)

24 Craig Perrin (craig.perrin@solent.ac.uk)

25 James Steele (James.steele@solent.ac.uk)

26 Jürgen Giessing (giessing@uni-landau.de)

27 Paulo Gentil (paulogentil@hotmail.com)

29 Word Count: 3873

30 Abstract: 297 
31

32

33

34

35

36

37

\section{Abstract}

Objectives: Common exercises such as the barbell back squat (BBS) and barbell hip thrust (BHT) are perceived to provide a training stimulus to the lumbar extensors. However, to date there have been no empirical studies considering changes in lumbar extension strength as a result of BBS or BHT resistance training interventions. Purpose: To consider the effects of barbell back squat (BBS) and barbell hip thrust (BHT) resistance training programmes upon isolated lumbar extension (ILEX) strength. Methods: Trained male subjects ( $\mathrm{n}=14 ; 22.07 \pm 0.62$ years; $179.31 \pm 6.96 \mathrm{~cm} ; 79.77 \pm 13.81 \mathrm{~kg}$ ) were randomised in to either BBS $(n=7)$ or BHT $(n=7)$ groups and performed 2 training sessions per week during a 4-week mesocycle using $80 \%$ of their 1RM. All subjects were tested pre- and post-intervention for BBS and BHT 1RM as well as isometric ILEX strength. Results: Analyses revealed that both BBS and BHT groups significantly improved both their BBS and BHT 1RM, suggesting a degree of transferability. However, the BBS group improved their BBS 1RM to a greater degree than the BHT group $(p=0.050 ; \sim 11.8 \mathrm{~kg} / 10.2 \%$ vs. $\sim 8.6 \mathrm{~kg} / 7.7 \%$, respectively). And the BHT group improved their BHT 1RM to a greater degree than the BBS group ( $p=0.034 ; \sim 27.5 \mathrm{~kg} / 24.8 \%$ vs. $\sim 20.3 \mathrm{~kg} / 13.3 \%$, respectively). Neither BBS nor BHT groups significantly improved their isometric ILEX strength. Conclusions: The present study supports the concept of specificity, particularly in relation to the movement mechanics between trunk extension (including pelvic rotation) and ILEX. Our data suggests that strength coaches, personal trainers, and trainees can self-select multi-joint lower body trunk extension exercises based on preference or variety. However, evidence suggests that neither the BBS nor BHT exercises can meaningfully increase isolated lumbar extension strength. Since strengthening these muscles might enhance physical and sporting performance we encourage strength coaches and personal trainers to prescribe ILEX exercise. 


\section{INTRODUCTION}

55

56

57

Low-back strength, particularly as a component part of core strength and stability, retains an importance in athletic performance and thus strength and conditioning within sports (Hibbs, et al. 2008). Indeed, the strength and cross-sectional area of the erector spinae and quadratus lumborum have been suggested to share $50 \%$ of the variance in sprint speed over $20 \mathrm{~m}$ (Kubo, et al. 2011), a substantial contribution for an inconspicuous muscle during sprinting. Furthermore, elevated forces through the lumbar spine whilst blocking in American football, as well as during a golf swing, suggest that improving lumbar strength might be beneficial towards both enhancing performance and reducing risk of injury (Gatt, et al. 1997; Gluck, et al. 2008). Finally, simulation research suggests erector spinae weakness results in compensation from synergistic muscles, potentially causing an earlier onset of fatigue and exacerbating low-back pain and performance decrements (Raabe and Chaudhari, 2018). Research has supported that common exercises such as the barbell back squat (BBS) place considerable stress on the lumbar musculature (Cholewicki, et al. 1991) supported by the high levels of activation of the lumbar muscles when measured by electromyography (EMG; Hamlyn, et al. 2007; Yavuz, et al. 2015). Indeed, as it is thought the lumbar extensor musculature is heavily involved in the BBS, many strength and conditioning coaches advocate the use of the barbell back squat (BBS) and deadlift exercises with a view to provide a training stimulus and increase the strength of the lumbar muscles (Mayer, et al. 2008).

Nevertheless, whilst the BBS exercise appears to be a hypothetical solution to strengthening the lumbar muscles (although no empirical evidence exists), alternative and perhaps superior exercises may be available. An exercise growing in popularity is the barbell hip thrust (BHT), which has been shown to produce greater EMG amplitude in the hamstring and gluteal muscles when compared to the BBS exercise (Contreras, et al. 2015). The BHT has also been shown to be associated with performance 
77

markers such as acceleration ( $r=0.93$; Loturco, et al. 2018). Furthermore, Contreras, et al. (2017)

recently compared 6-weeks of strength training using either a front squat or BHT exercise on performance markers in adolescent males. The authors reported favourable effect sizes for the BHT for $10 \mathrm{~m}$ and $20 \mathrm{~m}$ sprint times, and isometric mid-thigh pull, whereas the front squat exercise produced favourable effect sizes for the vertical jump and front squat 3RM. Andersen, et al. (2018) compared EMG activity during the barbell deadlift, hex bar deadlift and BHT exercises. Although there were no statistically significant differences between the three exercises for erector spinae muscle activation, the BHT did elicit the greatest muscle activation during the concentric phase of the movement when the hip angle was approaching or exceeding 180 degrees. The authors suggest that the BHT likely promotes increased muscle activation in the upper phase due to the increased hip torque requirement in the end range of this horizontally loaded exercise. In contrast, during a deadlift or BBS exercise the lumbo-pelvic complex reaches complete hip extension in a vertical anatomical position meaning the forces produced by the barbell load the skeletal system and likely produce lower muscular activation.

However, the BBS and BHT both involve the lumbo-pelvic complex in a compound movement integrating both hip- and lumbar-extension (i.e. trunk extension). Previous research considering both resistance machines (Graves, et al. 1994) and free-weight exercise (the Romanian deadlift; Fisher, et al. 2013) has suggested that trunk extension exercise (hip- and lumbar-extension) that permits rotation of the pelvis is not efficacious in increasing isolated lumbar extension strength. This is likely a result of hamstring and gluteal contribution via pelvic rotation, rather than isolated lumbar extension. Indeed, research has supported that there is significantly greater activation of the lumbar multifidus during back extension when the pelvis is stabilized (San Juan, et al. 2005), and in addition, muscle activation of the gluteus maximus and biceps femoris is decreased (Da Silva, et al. 2009). However, contrasting evidence does exist to support trunk extension tasks in producing isolated lumbar extension fatigue. For example, Edinborough, et al. (2016) reported that performing kettlebell swings produced transient fatigue in 
101 isolated lumbar extension (ILEX) strength, hypothesising that performing the kettlebell swing exercise as

102 part of a training programme might produce a chronic training effect.

Despite this, a recent study (Androulakis-Korakakis, et al. 2018) has reported that ILEX strength

104

105

106

107

108

109

110

111

112

113

114

115

116

117

118

119

120

121

122

123 does not differ between recreationally trained males and both competitive and non-competitive powerlifters; a population who regularly train with exercises such as the BBS. Currently reviews of the efficacy of different exercise approaches upon ILEX strength suggest that evidence is limited with respect to many approaches, yet that an ILEX exercise may be most efficacious (Steele, et al. 2015). However, devices for this are expensive, not readily available, and as such, the consideration of cheaper and accessible alternatives to ILEX exercise for strengthening the lumbar extensors should be considered.

To date, no empirical studies have assessed the efficacy of the either the BBS exercise or the BHT upon isolated lumbar extension strength. Thus, the aim of the present study was to consider the efficacy of a 4-week mesocycle of either BBS or BHT exercise in increasing ILEX strength.

\section{METHODS}

A randomised trial, research design was used whereby fourteen trained males were randomised in to either BBS $(n=7)$ or BHT $(n=7)$ training. Both groups were assessed pre- and post-intervention for BBS and BHT maximal strength (1-repetition maximum; 1RM) as well as isometric ILEX strength. The study was approved by Solent University Health, Exercise, and Sport Science (HESS) ethics committee (ID No. 669).

Using convenience and snowball sampling methods, fourteen trained males were recruited.

Subjects were required to have $>6$ months resistance training (RT) experience, and currently be performing a structured RT programme with at least one session per week including the use of BBS exercise and BHT exercise and have no history of low-back pain. Written informed consent was obtained

Peer] reviewing PDF | (2019:03:35781:2:0:NEW 12 Jun 2019) 
124 from all subjects prior to participation. Subjects were randomised using a computer randomisation

125 program to one of two groups; $\mathrm{BBS}(n=7)$, or $\mathrm{BHT}(n=7)$. Subjects were asked to refrain from any exercise

126 away from the supervised sessions. Participant demographics are included in table 1.

*INSERT TABLE 1 AROUND HERE*

A power analysis of previous research, using similar study design and asymptomatic subjects

(Contreras, et al. 2017; Styles, et al. 2016) was conducted to determine sample size (n). The effect size

130 (ES) was calculated for both studies using Cohen's $d$ (1992), producing a mean ES of 1.67 for BBS and

BHT strength increases. Participant numbers were calculated using equations from Whitley and Ball

(2002) revealing each group required 7 subjects to meet required $\beta$ power of 0.8 at an $\alpha$ value of $p<0.05$.

It should be noted that the study was powered for the identification of within group changes (two

tailed) in outcomes and between group comparisons were a secondary outcome. Based upon sensitivity

analysis for between group comparisons for the analysis detailed below the study was powered to identify at most a large between group effect size of $f=0.82$.

BBS and BHT exercises and verified their ability to perform them safely with correct technique. They also attended a familiarisation session for the MedX lumbar extension machine (MedX, Ocala, FL, USA) used

for at least 48 hours before baseline testing, and at least 48 hours before post-intervention testing. 
147 Maximum strength testing was consistent with recognized guidelines established by the NSCA (Baechle

148 and Earle, 2008). Prior to testing, subjects performed a general warm-up consisting of 5 minutes cycling

149 at 60-70 rpm and 50w. Next, a specific warm-up set of the prescribed exercise for 5 repetitions was

150 performed at $~ 50 \% 1$ RM followed by 1 to 2 sets of $2-3$ repetitions at a load corresponding to $60-80 \%$

151 1RM. Subjects then performed 1 repetition sets of increasing weight for 1RM determination. The

152 external load was adjusted by $\sim 5-10 \%$ in subsequent attempts until the subject was unable to complete

1531 maximal muscle action. The 1RM was considered the highest external load lifted. A 3- to 5-minute rest

154 was provided between each attempt. All 1RM determinations were made within 5 attempts. Test-retest

155 reliability was not determined within the present study, but instead was calculated from previous

156 studies as the typical error of measurement and 95\%Cls. For the BBS, this was calculated as $3.8 \mathrm{~kg}$

$157(95 \% \mathrm{Cls}=2.9$ to $5.5 \mathrm{~kg})$ from the average of typical errors of measurement (using average of participant

158 sample sizes to determine degrees of freedom for calculation of $95 \% \mathrm{Cls}$ ) reported in studies reviewed

159 on BBS 1RM reliability by Nuzzo et al. (2019) with a range of 2-4 days between test-retest session. For

160 the $\mathrm{BHT}$, this was calculated as $8.3 \mathrm{~kg}(95 \% \mathrm{Cls}=5.7$ to $15.2 \mathrm{~kg})$ from the standard deviation of changes

161 from the control group reported by Bishop et al. (2017) over a period of 8 weeks.

The BBS 1RM was completed first, followed by the BHT 1RM, with a 20-minute rest interval

between exercises to allow for sufficient recovery. As per Contreras, et al. (2015), the BHT was

performed by having subjects' upper back on a bench with the feet positioned wider than shoulder

width and toes pointed forwards or slightly outwards. The barbell was padded with a thick bar pad and placed over the subjects' hips. Subjects were instructed to thrust the bar upwards while maintaining a neutral spine and pelvis. Full extension of the hips $\left(180^{\circ}\right)$ was required for a successful lift. 
171 tightly to ensure the effort produced was from the lumbar musculature only and not from the pelvis or

172 thighs, isolating the lumbar extensors. A counter-weight was used to balance the mass of the upper

173 body and the effects of gravity on the upper body. All subjects were assessed for range of motion (ROM)

174 and performed a dynamic warm-up with a load equating to $90 \mathrm{lbs} / \sim 41 \mathrm{~kg}$ and three submaximal

175 isometric tests at full flexion, full extension and a mid-range position. Maximal isometric testing was

176 then performed at 7 joint angles $\left(0^{\circ}, 12^{\circ}, 24^{\circ}, 36^{\circ}, 48^{\circ}, 60^{\circ}\right.$ and $72^{\circ}$ of extension) where subjects were

177 encouraged to gradually achieve maximal effort over 2-3 seconds and to maintain the maximal

178 contraction for a further 1 second. The torque produced was measured by a load cell attached to the

179 movement arm. Subjects rested for 5-10 seconds between tests at different joint angles. ILEX strength

was considered as a 'strength index' (SI) calculated as the area under the torque curve (using the

trapezoid formula) from multiple angle testing in order to provide a composite measure of overall

changes in strength across the range of motion (see supplementary file). Based upon between day

repeated test-retest data from prior studies in our lab (Edinburough, et al. 2016; Stuart, et al. 2018) we

determined the typical error of measurement for the SI as $1659.51 \mathrm{Nm} \cdot$ degrees $(95 \% \mathrm{Cls}=1316.46$ to

2317.17 Nm·degrees) using Hopkins (2015) spreadsheets for reliability.

Both the BBS and BHT participant groups attended 2 resistance training session per week, for a

4-week mesocycle. During these sessions each participant performed 3 sets using $80 \%$ of their 1 RM

( mean=8RM; range=6-10RM) in a controlled, non-explosive manner ( 2 seconds concentric, 4 seconds 
195

196

197

198

199

200

201

202

203

204

205

206

207

208

209

210

211

212

213

214

215

216

217

218

sets. All training sessions were supervised one-to-one and attempts were made to increase the load lifted each week whilst maintaining the target repetition range. No injuries were reported and adherence to the programme was $100 \%$ for both groups.

The independent variable was the group (BBS or $\mathrm{BHT}$ ) and dependent variables changes (i.e. post-test minus pre-test values) in BBS 1RM, BHT 1RM, and SI. Analysis of covariance (ANCOVA) was used for between group comparisons in dependent variables with baseline measures as covariates (i.e., pre-intervention BBS 1RM, BHT 1RM, and SI). Point estimates were calculated along with the precision of those estimates using $95 \%$ confidence intervals $(\mathrm{Cl})$ for within group adjusted means. The $95 \% \mathrm{Cls}$ were further interpreted to indicate that significant within group changes occurred if the upper or lower limits do not cross zero. The software used was SPSS (version 23, IBM Corp, Portsmouth, UK) and the cut off for significance was $p<0.05$. Gardner-Altman plots were also produced using Estimation Statistics (Claridge-Chang and Assam, 2016) for data visualization. Visual inspection of the data using boxplots revealed 2 outliers (determined using the interquartile rule) for both change in BBS 1RM (in the BHT group) and change in BHT 1RM (in the BBS group) and further Shapiro-Wilk tests confirmed that data did not meet assumptions of normality of distribution for the groups containing the outliers (change in squat 1RM, BHT group $p<0.001$; change in BHT 1RM, BBS group $p<0.001$ ). Thus, for these variables, due to the significant deviations from normality of distribution combined with the relatively small sample size, the data were rank transformed prior to performing ANCOVA (Olejnik, et al. 1984). All results are reported in the units of measurement for each test.

\section{RESULTS}

The $95 \% \mathrm{Cls}$ for changes did not cross zero for change in squat 1RM or change in BHT 1RM in either group and thus both groups had significant within-group improvements in these outcomes which also exceeded the typical errors of measure. The $95 \% \mathrm{Cls}$ for changes in SI did not cross zero for the BHT group suggesting a significant change; however, change in SI did not exceed typical error of 
219 measurement for either group and so is unlikely to be a meaningful change. Between group

220 comparisons using ANCOVA revealed significant differences for change in squat 1 RM $(F(1,11)=5.240, p=$

$2210.043)$, change in $\operatorname{BHT} 1 \operatorname{RM}\left(F_{(1,11)}=6.673, p=0.025\right)$, but not for change in SI $\left(F_{(1,11)}=1.541, p=0.240\right)$.

222 Table 2 shows pre- and post-intervention results, unadjusted means ( \pm SD) or medians ( \pm IQR) for rank

223 transformed variables for changes in each outcome measure, and 95\%Cls for the changes. For visual

224 depiction of results, Gardner-Altman plots of changes for each outcome are presented in figure 2.

*INSERT FIGURE 2 AROUND HERE*

226

*INSERT TABLE 2 AROUND HERE*

227

228

229

230

231

232

233

234

235

236

237

\section{DISCUSSION}

To the authors knowledge this is the first study to investigate the effects of BBS and BHT strength training upon ILEX strength. As such this research adds to a dearth of literature considering exercise protocols to improve lumbar extension strength.

We should first consider the efficacy of the training routine for the specific exercises. Our analyses revealed that the BBS training group significantly improved BBS and BHT 1RM by averages of $\sim 11.8 \mathrm{~kg} / 10.2 \%$, and $\sim 20.3 \mathrm{~kg} / 13.3 \%$, respectively. Furthermore, the BHT training group significantly improved BBS and BHT 1RM by averages of $\sim 8.6 \mathrm{~kg} / 7.7 \%$ and $\sim 27.5 \mathrm{~kg} / 24.8 \%$, respectively. Further, these changes exceeded the typical errors of measurement. Whilst specificity meant that both groups improved their strength to a significantly greater degree on the exercise they used during training (e.g. the BBS group improved their BBS 1RM to a greater degree than the BHT group, and the BHT group improved their BHT 1RM to a greater degree than the BBS group), the present data suggests a degree of transferability between exercises. In considering transferability, it is noteworthy that the largest individual strength increase in BBS $1 \mathrm{RM}(25 \mathrm{~kg})$ was a participant in the BHT group, and the largest individual increase in BHT 1RM $(55 \mathrm{~kg})$ was a participant in the BBS group. Whilst our data cannot explain

Peer] reviewing PDF | (2019:03:35781:2:0:NEW 12 Jun 2019) 
242 individual differences based on the possible heterogeneity of the participant group, we might reflect on

243 the degree of training status of these participants that accommodates such large strength increases over

244 a 4-week training cycle. Any attempt at explaining these large individual changes would be purely

245 speculative, however, we postulate that they might have arisen from the mesocycle prior to this

246 intervention period not being focused on maximal strength, and/or that participants might have

247 benefited from direct individual supervision, as has been seen in previous research with some training

248 experience (Coutts, et al. 2004). As such the participants in both the BBS and BHT group might never

249 have trained to the same intensity of effort and as such made significant strength increases which

250 showed transferability. In addition the participant in the BHT group might have benefited from practice

251 of recruiting the gluteal muscles which, in turn, supported improvement in the BBS 1RM. For example

252 previous research by Crow, et al. (2012) supports acute improvements in counter-movement jump

253 following specific gluteal exercises. It might be that there are chronic adaptations to multi-joint lower

254 body movements that support the targeting of the gluteal muscle group as part of a training

255 intervention.

However, our data suggests that neither the BBS nor BHT resistance training exercises serve to

improve ILEX strength, since the pre- to post-intervention changes did not exceed the typical error of

258

259

260

261

262

263

264

265 measurement. Recent research serves to support our findings in context of the BBS exercise. For example, Vigotsky, et al. (2018), recently reported no relationship between BBS 1RM and isometric spinal extension strength. In their discussion, Vigotsky, et al. suggested that, during a BBS exercise, "the spinal erectors need only resist the net joint moment as well as a small abdominal co-contraction, which does not necessarily increase with load". Additionally, Androulakis-Korakkakis, et al. (2018) recently reported isometric lumbar extension torque and BBS 1RM values for non-competitive powerlifters (NCPL) and competitive powerlifters (CPL). The data suggested that, despite large and significant differences in BBS 1RM (NCPL=177.0kg, CPL=215.2kg), there were no differences in ILEX strength (SI; 
$266 \mathrm{NCPL}=22864 \mathrm{Nm} \cdot$ degrees, $\mathrm{CPL}=22850 \mathrm{Nm} \cdot$ degrees). Indeed, data from the present study produced

267 similar ILEX strength values 20000-21820 Nm·degrees supporting that beyond a certain threshold the

268 lumbar extensors might not be required to increase in strength to aid BBS performance. However,

269 despite the lack of association between ILEX strength and BBS strength, and the existence of an

270 association between performance markers and the BBS, it is not wholly clear whether increasing lumbar

271 extension strength through isolated training might also increase performance. The absence of specific

272 isolated lumbar extensor training may be suboptimal for developing athletic performance. Indeed,

273 Fisher et al. (2013) have shown that ILEX resistance training can increase Romanian deadlift 1RM.

274 Further research is required though to examine ILEX training interventions upon performance outcomes,

275 including BBS strength and sporting performance.

The BHT represents a more contemporary, and thus limited, area of exercise science research.

277 Certainly evidence has suggested that the BHT might be an efficacious exercise for improving sprint performance and mid-thigh pull (Contreras, et al. 2017), and data supports considerable muscle activation of the hamstring and gluteal muscles (Contreras, et al. 2015). In addition, research has suggested greater erector spinae muscle activation for the BHT compared to the barbell- and hex bardeadlifts (Andersen, et al. 2018), and as noted improving ILEX strength can serve to improve Romanian deadlift 1RM (Fisher, et al. 2013). However, the present study suggests that training, and indeed enhancing 1RM for the BHT exercise does not improve ILEX strength. This specificity of adaptation is further supported as Fisher, et al. (2013) also reported a group training using the Romanian deadlift increased their Romanian deadlift 1RM significantly but failed to increase their ILEX strength. 
290 be that the gluteal and hamstring muscles serve to provide trunk-extension (e.g. hip- and lumbar-

291 extension), and as a result both BBS and BHT resistance training produce strength increases in these

292 muscles which can transfer to improve performance between the respective exercises. However, as a

293 result of the pelvic rotation through trunk extension (and thus the dominance of the gluteal and

294 hamstring muscles), neither BBS nor BHT exercises appear to provide a sufficient training stimulus to the

295 lumbar extensors. This is fitting with previous research which has reported no relationship between

296 trunk extension performance (assessed via Biering-Sorensen test) and isolated lumbar extension

297 strength in both asymptomatic persons as well as those symptomatic with chronic low back pain

298 (Conway, et al. 2017). Nonetheless, some tasks performed with pelvic rotation can induce lumbar

299 fatigue suggesting a role for this muscle during movement, for example kettlebell swings (Edinborough,

300 et al. 2016). Though the present data suggests that both the BBS and BHT do not improve lumbar

301 extension strength, and previous data suggests the Romanian deadlift also lacks efficacy (Fisher, et al.

302 2013), further research should consider other exercises such as the kettlebell swings in training

303 interventions.

304

We should, of course, remember that exercises such as the BBS are not performed solely with

305

the intent of strengthening the lumbar extensors, and that this exercise shows a strong relationship to

athletic performance markers such as sprint speed $(r=0.71-0.94)$ and vertical jump ( $r=0.78$; Wisløff, et al.

2004). However, a previous review has questioned the need for adding single-joint exercise to a

resistance training programme since muscular adaptations appear similar to when performing only

multi-joint exercises (Gentil, et al. 2017). As mentioned above we might consider trunk extension (e.g.

hip- and lumbar-extension) to be multi-joint, and isolated lumbar extension to be more similar to single-

311 joint movements (though strictly speaking it is a multi-joint movement due to the vertebral segments).

312 However, in light of the previous research as well as present findings, it appears that multi-joint

313 exercises which include trunk extension (such as the BBS and BHT) are not sufficient to strengthen the

Peer] reviewing PDF | (2019:03:35781:2:0:NEW 12 Jun 2019) 
314 lumbar extensors. As such, though multi-joint movements may be sufficient for appendicular muscular

315 adaptations (Gentil, et al. 2017), we would suggest that both athletes and lay persons consider

316 supplementing existing training practices with specific ILEX exercise to strengthen the lumbar muscles.

Whilst the present study provides useful data and guidance as to the efficacy of different

exercises in strengthening the lumbar extensors, we should accept the limitation that we did not include an ILEX training group which might reflect the possible comparative increases in ILEX strength. Previous research has demonstrated that isometric ILEX strength can increase considerably as a result of once weekly training sessions using $80 \%$ MVC over 10-weeks (strength index change=4353 Nm-degrees; Fisher, et al. 2013). Furthermore, interventions performed once a week over 6 week durations have been shown to produce significant increases in lumbar extension strength using both single- and multiple-sets in trained males (single set $=1854 \mathrm{Nm} \cdot$ degrees, multiple set $=2415 \mathrm{Nm} \cdot$ degrees; Steele, et al. 2015), and with both heavier-loads ( $80 \% \mathrm{MVC})$ and lighter-loads (50\% MVC) in recreationally active males and females (strength index change; 80\% MVC = $2891 \mathrm{Nm} \cdot$ degrees, 50\% MVC = $2865 \mathrm{Nm} \cdot$ degrees; Fisher, et al. 2018). As such, had we included an ILEX training group, we would expect 8 sessions performed over 4 weeks to have likely produced significant and meaningful increases in isometric ILEX strength. A further limitation might be the brevity of the present 4-week strength mesocycle. Whilst we contest that this is fitting with training practices, we accept that many athletes and persons undertake longer mesocycles, or continue exercises across multiple phases of periodisation. Future research might consider the transference of adaptations as a result of continued BBS or BHT resistance training through strength, power and/or hypertrophy loading phases. Lastly, this study was relatively small and primarily powered to identify within group changes in outcomes. Though we did identify between group differences in both BBS and BHT 1RM changes future research with greater sample sizes should examine 
338 might be that analyses of individual angles would reveal angle specific strength increases - particularly

339 at $0^{\circ}$ (full extension) where the hip thrust exercise might have provided the greatest activation of the

340 lumbar extensors (e.g. at $180^{\circ}$ during a BHT exercise; Andersen, et al. 2018). Future research might

341 consider sufficient sample sizes and power to examine this as a pre-specified hypothesis.

\section{CONCLUSION}

The present study provides support for the concept of specificity, particularly in relation to the movement mechanics between trunk extension (including pelvic rotation) and isolated lumbar extension. Our data suggests that both the BBS and BHT exercises produce meaningful increases in strength which might transfer between lower-body trunk extension exercises. This allows strength coaches, personal trainers, and trainees to self-select multi-joint lower body trunk extension exercises based on preference or variety. However, evidence suggests that neither the BBS nor BHT exercises, nor indeed any exercise allowing pelvic rotation through trunk extension, can meaningfully increase ILEX strength. Since strengthening these muscles might enhance physical and sporting performance we encourage strength coaches and personal trainers to supplement existing practices by prescribing

352 specific ILEX exercise. 


\section{REFERENCES}

354 Andersen, V, Fimland, MS, Mo, DA, Iversen, VM, Vederhus, T, Rockland Helleb $\varnothing$, LR, Nordaune, KI, and 355 Saeterbakken, AH. Electromyographic Comparison of Barbell Deadlift, Hex Bar Deadlift and Hip Thrust 356 Exercises: A Cross-Over Study. J Strength Cond Res 32(3): 587-593, 2018.

357 Androulakis-Korakakis, P, Gentil, P, Fisher, JP, and Steele, J. Comparison of isolated lumbar extension 358 strength in competitive and non-competitive powerlifters, and recreationally trained males. J Strength 359 Cond Res. Epub ahead of print, 2018.

360 Baechle, TR, and Earle, RW. Essentials of strength training and conditioning. Champaign, IL: Human 361 Kinetics, 2008.

362

363

Bishop, C, Cassone, N, Jarvis, P, Turner, A, Chavda, S, and Edwards, M. Heavy Barbell Hip Thrusts Do Not Effect Sprint Performance: An 8-Week Randomized-Controlled Study. J Strength Cond Res. Epub ahead of print, 2017.

Cholewicki J, McGill SM, and Norman RW. Lumbar spine loads during the lifting of extremely heavy weights. Med Sci Sports Exerc 23(10): 1179-1186, 1991.

Claridge-Chang, A, and Assam, PN. Estimation statistics should replace significance testing. Nat Methods 13(2): 108-109, 2016.

Cohen, J. A power primer. Psychol Bull 112: 155-159, 1992.

Contreras, B, Vigotsky, AD, Schoenfeld, BJ, Beardsley, C, and Cronin, J. A Comparison of Gluteus Maximus, Biceps Femoris, and Vastus Lateralis Electromyographic Activity in the Back Squat and Barbell Hip Thrust Exercises. J Appl Biomech 31(6): 452-458, 2015.

Contreras, B, Vigotsky, AD, Schoenfeld, BJ, Beardsley, C, McMaster, DT, Reyneke, JH, and Cronin, JB. Effects of a six-week hip thrust vs. Front squat resistance training program on performance in adolescent males: a randomized controlled trial. J Strength Cond Res 31(4): 999-1008, 2017.

Conway, R, Behennah, J, Fisher, J, Osborne, N, and Steele, J. Associations between trunk extension endurance and isolated lumbar extension strength in both asymptomatic participants and those with chronic low back pain. Healthcare 4(3); pii E70, 2017.

Crow, JF, Buttifant, D, Kearny, SG, and Hrysomallis, C. Low load exercises targeting the gluteal muscle group acutely enhance explosive power output in elite athletes. J Strength Cond Res 26(2): 438-442.

Coutts, AJ, Murphy, AJ, and Dascombe, BJ. Effect of direct supervision of a strength coach on measures of muscular strength and power in young rugby league players. J Strength Cond Res 18(2): 316-323.

Da Silva, RA, Lariviere, C, Arsenault, AB, Nadeau, S, and Plamondon, A. Pelvic stabilization and semisitting position increase the specificity of back exercises. Med Sci Sports Exerc 41(2): 435-443, 2009.

Day, ML, McGuigan, MR, Brice, G, and Foster, C. Monitoring exercise intensity during resistance training using the session RPE scale. J Strength Cond Res 18(2): 353-358, 2004. 
387

388

389

390

391

392

393

394

395

396

397

398

399

400

401

402

403

404

405

406

407

408

409

410

411

412

413

414

415

416

417

418

419

Edinborough, L, Fisher, JP, and Steele, J. A comparison of the effect of kettlebell swings and isolated lumbar extension training on acute torque production of the lumbar extensors. J Strength Cond Res 30(5): 1189-1195, 2016.

Fisher, J, Bruce-low, S, and Smith, D. A randomized trial to consider the effect of Romanian deadlift exercise on the development of lumbar extension strength. Phys Ther Sport 14(3): 139-145, 2013.

Fisher, JP, Stuart, C, Steele, J, Gentil, P, and Giessing, J. Heavier- and lighter-load isolated lumbar extension resistance training produce similar strength increases, but different perceptual responses, in healthy males and females. PeerJ, 6:e6001, 2018.

Gatt CJ, Hosea TM, Palumbo, RC, and Zawasky, JP. Impact Loading of the Lumbar Spine during Football Blocking. Am J Sports Med 25(3): 317-321, 1997.

Gentil, P, Fisher, J, and Steele, J. A review of the acute effects and chronic adaptations of single- and multi-joint exercises during resistance training. Sports Med 47(5):843-855, 2017.

Gluck, GS, Bendo, JA, and Spivak, JM. The lumbar spine and low back pain in golf: a literature review of swing biomechanics and injury prevention. Spine J 8:778-788, 2008.

Graves, JE, Pollock, ML, Leggett, SH, Carpenter, DM, Vuoso, RM, and Jones, A. Effects of training Frequency and Specificity on Isometric Lumbar Extension Strength. Spine 15: 289-294, 1990.

Graves, JE, Webb, DC, Pollock, ML, Matkozich, J, Leggett, SH, Carpenter, DM, Foster, DN, and Cirulli, J. Pelvic stabilisation during resistance training: its effect on the development of lumbar extension strength. Arch Phys Med Rehabil 75(2): 210-215, 1994.

Hamlyn, N, Behm, DG, and Young, WB. Trunk muscle activation during dynamic weight-training exercises and isometric instability activities. J Strength Cond Res 21(4): 1108-1112, 2007.

Hibbs, AE, Thomspon, KG, French, D, Wrigley, A, and Spears, I. Optimizing Performance by Improving Core Stability and Core Strength. Sports Med 38(12): 995-1008, 2008.

Hopkins WG. Spreadsheets for analysis of validity and reliability. Sportscience 19: 36-42, 2015.

Kubo, T, Hoshikawa, Y, Muramatsu, M, lida, T, Komori, S, Shibukawa, K, and Kanehisa, H. Contribution of trunk muscularity on sprint run. Int J Sports Med 32(3): 223-228, 2011.

Loturco, I, Contreras, B, Kobal, R, Fernandes V, Moura, N, Siqueira, F, Winckler, C, Suchomel, T, and Pereira, LA. Vertically and horizontally directed muscle power exercises: Relationships with top-level sprint performance. PLoS One 13(7): e0201475, 2018.

Mayer, J, Mooney, V, and Dagenais, S. Evidence-informed management of chronic low back pain with lumbar extensor strengthening exercises. Spine J 8(1): 96-113, 2008.

Nuzzo, JL, Taylor, JL, and Gandevia, SC. CORP: Measurement of upper and lower limb muscle strength and voluntary activation. J Appl Physiol 126(3): 513-543, 2019. 
420 Olejnik, SF, and Algina, J. Parametric ANCOVA and the Rank Transform ANCOVA When the Data are

421 Conditionally Non-Normal and Heteroscedastic. J Educ Behav Stat 9(2): 129-149, 1984.

422 Pollock ML, Leggett, SH, Graves, JE, Jones, A, Fulton, M, and Cirulli, J. Effect of Resistance Training on

423 Lumbar Extension Strength. Am J Sports Med 17: 624-629, 1989.

424 Raabe, M, and Chaudhari, AMW. Biomechanical consequences of running with deep core muscle

425 weakness. J Biomech 7: 98-105, 2018.

426 San Juan, JG, Yaggie, JA, Levy, SS, Mooney, V, Udermann, BE, and Mayer, JM. Effects of pelvic

427 stabilization on lumbar muscle activity during dynamic exercise. J Strength Cond Res 19(4): 903-907,

4282005.

429 Steele, J, Bruce-Low, S, and Smith, D. A review of the specificity of exercises designed for conditioning

430 the lumbar extensors. Br J Sports Med 49(5): 291-297, 2015.

431 Steele, J, Fitzpatrick, A, Bruce-Low, S, and Fisher, J. The effects of set volume during isolated lumbar

432 extension resistance training in recreationally trained males. PeerJ 3:e878, 2015.

433 Steele. J, Fisher, J, Giessing, J, and Gentil, P. Clarity in Reporting Terminology and Definitions of Set End

434 Points in Resistance Training. Muscle Nerve 56(3): 368-374, 2017.

435 Stuart, C, Steele, J, Gentil, P, Giessing, J, and Fisher, JP. Fatigue and perceptual responses of heavier- and 436 lighter-load isolated lumbar extension resistance exercise in males and females. PeerJ 6: e4523, 2018.

437 Styles, WJ, Matthews, MJ, and Comfort, P. Effects of Strength Training on Squat and Sprint Performance 438 in Soccer Players. J Strength Cond Res 30(6): 1534-1539, 2016.

439 Vigotsky, AD, Bryanton, MA, Nuckols, G, Beardsley, C, Contreras, B, Evans, J, and Schoenfeld, BJ.

440 Biomechanical, anthropometric, and psychological determinants of barbell back squat strength. J

441 Strength Cond Res E-Pub ahead of print, 2018.

442 Whitley, E, and Ball, J. Statistics review 4: sample size calculations. Crit Care 6: 335-341, 2002.

443 Wisløff U, Castagna C, Helgerud J, Jones R, and Hoff J. Strong correlation of maximal squat strength with 444 sprint performance and vertical jump height in elite soccer players. Br J Sports Med 38(3): 285-288, 4452004.

446 Yavuz, HU, Erdağ, D, Amca, AM, and Aritan, S. Kinematic and EMG activities during front and back squat 447 variations in maximum loads. J Sports Sci 33(10): 1058-1066, 2015. 
Figure 1

MedX Lumbar Extension Machine showing restraint system

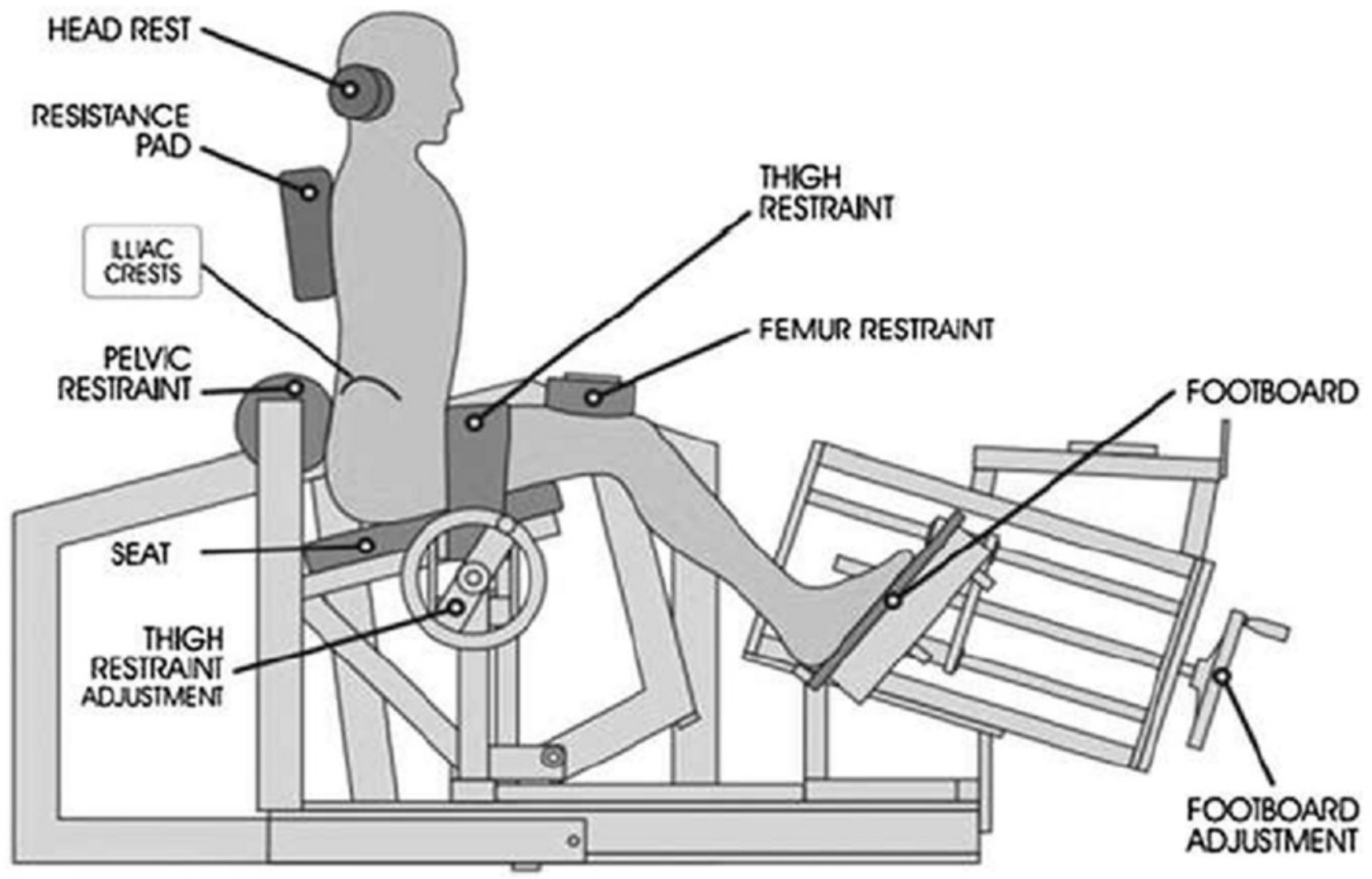


Figure 2

Gardner-Altman Plots - all participants

Figure shows changes for each outcome for all participants 

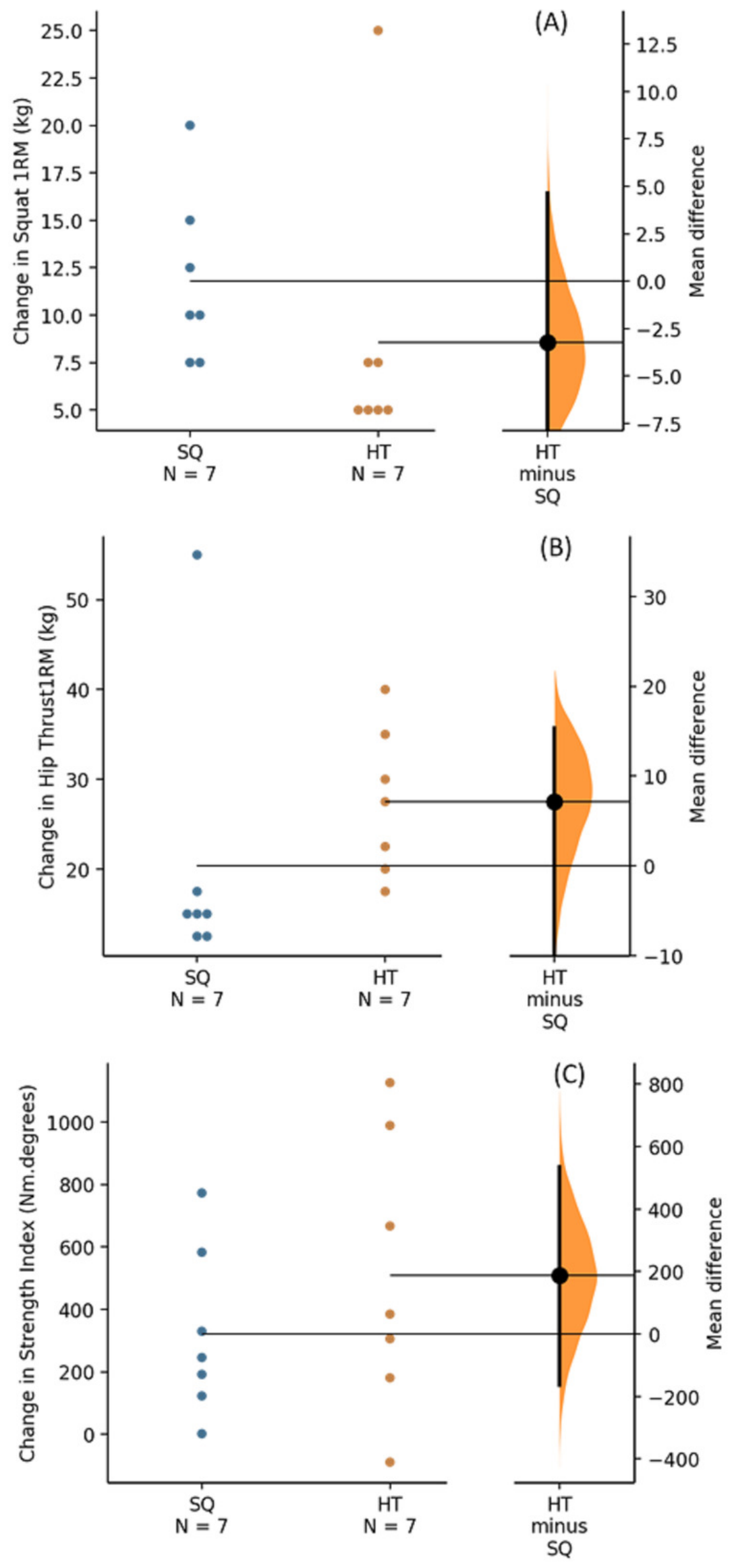

Peer] reviewing PDF | (2019:03:35781:2:0:NEW 12 Jun 2019) 
Table $\mathbf{1}$ (on next page)

Participant Characteristics 


\begin{tabular}{l}
1 Table 1. Participant Characteristics \\
$\qquad \begin{array}{ccc} \\
3\end{array}$ \\
\cline { 2 - 3 } \\
\begin{tabular}{ccc} 
Characteristic & Back Squat $(n=7)$ & Barbell Hip Thrust $(n=7)$ \\
\hline Age (years) & $21.71 \pm 0.45$ & $22.43 \pm 0.49$ \\
Height (cm) & $181.61 \pm 4.63$ & $177 \pm 7.61$ \\
Body mass (kg) & $76.49 \pm 9.09$ & $83.06 \pm 15.81$ \\
\hline
\end{tabular}
\end{tabular}

4 


\section{Table 2 (on next page)}

Pre- and Post-intervention 1-repetition maximum (1RM; means \pm SD) and isolated lumbar extension strength ( $\mathrm{N} \cdot \mathrm{m})$, changes, and 95\% confidence intervals (Cls)

Data for back squat 1RM and hip thrust $1 R M$ are presented as medians ( $\pm I Q R)$, whereas data for isolated lumbar extension strength is presented as mean $( \pm \mathrm{SD})$ 
Table 2. Pre- and Post-intervention 1-repetition maximum (1RM) and isolated lumbar extension strength (strength index; $\mathrm{SI}$ ), changes, and $95 \%$ confidence intervals (Cls)

\begin{tabular}{|c|c|c|c|c|c|}
\hline Group & Variable & Pre-intervention & Post-intervention & Changes & $95 \% \mathrm{Cls}$ for change \\
\hline \multirow[t]{5}{*}{ Back squat group ( $n=7)$} & Back Squat 1RM (kg) & $115.0 \pm 35.0$ & $122.5 \pm 27.5$ & $10.0 \pm 7.5$ & 7.6 to 15.9 \\
\hline & Hip Thrust 1RM (kg) & $160.0 \pm 42.5$ & $180.0 \pm 50.0$ & $15.0 \pm 5.0$ & 6.1 to 34.6 \\
\hline & Isolated Lumbar & $19670 \pm 1974$ & $20000 \pm 1847$ & $321 \pm 270$ & 25 to 600 \\
\hline & Extension Strength & & & & \\
\hline & (Nm·degrees) & & & & \\
\hline \multirow[t]{5}{*}{ Hip Thrust group ( $n=7)$} & Back Squat 1RM (kg) & $110.0 \pm 7.5$ & $117.5 \pm 17.5$ & $5 \pm 2.5$ & 1.8 to 15.4 \\
\hline & Hip Thrust 1RM (kg) & $162.5 \pm 25.0$ & $187.5 \pm 42.5$ & $27.5 \pm 15.0$ & 19.9 to 35.1 \\
\hline & Isolated Lumbar & $21310 \pm 2950$ & $21820 \pm 2998$ & $509 \pm 439$ & 231 to 856 \\
\hline & Extension Strength & & & & \\
\hline & (Nm·degrees) & & & & \\
\hline
\end{tabular}

5

Note: Data for back squat $1 R M$ and hip thrust $1 R M$ are presented as medians ( $\pm I Q R)$, whereas data for isolated lumbar extension strength is presented as mean $( \pm S D)$ 